\title{
Great leap outwards
}

Ideas in science can become so familiar that we no longer see them clearly. Their form comes to look final, and seemingly impervious to further improvement or change. Take the basic logic of Darwinian evolution, for example. We can easily imagine future refinements to include phenomena such as epigenetics or horizontal gene transfer, but the core ideas - genetic inheritance coupled with variation and natural selection - look fixed. Surely, nothing as fundamental as these ideas remains to be discovered?

But perhaps we are wrong. Perhaps we're just too familiar with what we know to see the possibilities. That idea is raised in provocative recent work by Teppo Felin and colleagues, which probes at the boundaries of physics, philosophy and biology, focusing on the puzzling nature of innovation - how true, unprecedented novelty emerges in biology, economics, technology or human culture (T. Felin et al. Strategic Entrepreneur. J.; in the press). Obviously, innovation requires the creation of something that did not exist before. It's less obvious, Felin et al. argue, that we understand much about the process, or currently even use the right mathematics to think about it.

As they point out, a key metaphor underlying much modern thinking about evolution, or the development of technology, is that of exploration within a complex or rugged multidimensional fitness landscape. The dynamics of evolution involve populations climbing the hills of higher fitness, their progress hampered by myriad constraints and trade-offs; they often get hung up on local peaks, unable to reach higher peaks further away. Discussions of technological or cultural evolution often rest on similar metaphors of searching for good solutions within a complex design space.

Innovation, in any such view, comes about from the discovery of previously inaccessible, hard-to-find peaks on such a landscape, perhaps following a long search, or in the wake of some destabilizing episode of change that riles the landscape and opens up new paths. But importantly, such metaphors always view the space of possible designs as something given or defined in advance. Search takes place within a fixed space, looking for currently unexplored parts of it.

In this sense, innovation occurs in much the same way that a physical system - say,

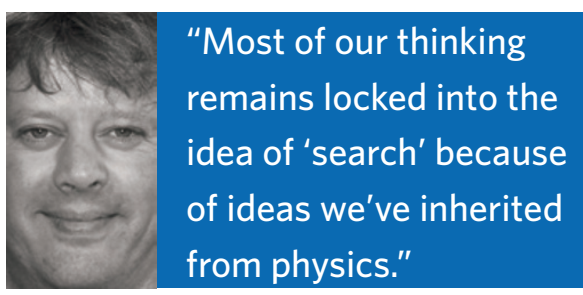

the atoms in a crystal - explores its possible configurations over time. There's a defined space and an algorithmic search over it. Felin and colleagues argue that this isn't enough; this perspective doesn't give innovation proper respect. In biology, technology, in mathematics or science itself, innovation is often much more than just an algorithmic search over existing possibilities; it's invention of altogether new possibilities, and possibly not algorithmic at all.

In the setting of technology, for example, would it even be possible to list all of the conceivable future innovations? Not just humanly possible, but mathematically? Felin et al. argue that such a list is not possible even in principle. Indeed, they suggest, it's not even possible to make a list of all conceivable applications of simple things that already do exist.

Take a screwdriver: the possible uses for it clearly include the obvious ones, plus an infinite set of non-obvious ones (as an electrical conductor, as a tool for cooling drinks or for the precision-sorting of small stones, as a weapon, an optical reflector and so on) plus another infinite set of uses that are absolutely impossible to imagine because they involve interaction with future objects, technologies and situations that do not yet exist.

The set of possible innovations isn't likely to be 'prestatable' or 'listable' in any way whatsoever. Hence, innovation must often (but not always) involve creation that cannot be achieved by a methodical mechanical search or any computable process acting on a fixed space of possibilities. It requires truly creative leaps outside of any such space. As the authors put it: "No algorithm can pre-decide what to do in novel environments whose nature cannot be prestated (at the relevant level of specificity). But the mind is generative and perhaps somehow alert to new possibilities in the adjacent possible. The mind is not algorithmic; no set of entailing laws can describe it."
Unfortunately, they note, most of our thinking remains locked into the idea of 'search' because of ideas we've inherited from physics - useful ideas, in their own setting, but inappropriate for thinking about innovation. In physics, after all, it has been commonplace for a century and a half to study the dynamics of any system using the concept of a phase space - that is, a space of possible states. Whether it is a simple dynamical system such as a pendulum, or a more complex one such as a galaxy of gravitationally interacting stars or a disordered glass, the space of states can be mathematically defined in specific form.

This way of thinking has spilled out across the sciences. It's implicit in the landscape metaphor of biology, in ecology, and has even been adopted in fields such as economics. For example, financial economists or macro-economists build mathematical theories on the assumption that it is meaningful to talk about a space of possible 'states of nature' possible outcomes at any moment in the future - determined by some probability distribution. Unfortunately, this renders the wild, 'unprestatable' uncertainty of the future rather benign; it creates an illusion of tidy simplicity where it does not belong. It's not going too far to suggest that this illusion played a role in creating wide complacency in mathematical economics before the recent financial crisis.

So Felin and colleagues' way of thinking looks promising to me, even if it is very preliminary. Their paper is a prescient signpost pointing towards things we don't yet understand or don't quite have the language to describe, in economics, evolutionary biology and elsewhere. As they note, acknowledging that the future unfolds into a space of unprestatable possibilities might seem like giving in to unrestricted randomness. But the paths of evolution, or of economic and technological innovation, aren't like that either; the randomness is of a particular kind, highly 'canalized'. The set of directions heading into the 'adjacent possible' is huge, but it is not literally infinite.

We need very unfamiliar ideas - new mathematics and new concepts that can help us define and perceive 'the topology of the possible'.

MARK BUCHANAN 